\title{
Delayed perforated ulceration at the entero-enteric anastomosis after rouex en-y gastric bypass
}

\begin{abstract}
Marginal ulceration after gastric bypass is a proven complication with an incidence between $1 \%$ and $16 \%$, and the exact mechanism of ulceration is not well established. Helicobacter pylori, smoking, Non-Steroidal Anti-inflammatory Drug (NSAID) use, and noncompliance with Proton Pump Inhibitor (PPI) postoperatively may contribute to marginal ulceration, but evidence is still lacking in the literature. This report reviews the case of a 25-year-old woman who underwent a combined Roux-en-Y gastric bypass after a Laparoscopic Sleeve Gastrostomy (LSG) for morbid obesity and subsequently developed a delayed perforated ulceration at the entero-enteric anastomosis post Rouex en-Y gastric bypass. In the literature, there are few examples of cases similar to our patient's case with explanations of the predisposing factors, presentation, and management of the ulceration at the anastomosis post Rouex en-Y gastric bypass.
\end{abstract}

Keywords: rouex en-y, gastric bypass, marginal ulcer, perforation, laparoscopy

\author{
Volume 6 Issue 5 - 2017
}

Al Hunaidi M,' Al Haddad M,' Al Subaie S,' Al Qinai S,' Alkhuder T,' Lourero M $^{2}$

'Department of surgery, Amiri Hospital, Kuwait

'IJP Cirurgia Mini Invasiva, Brazil

Correspondence: Al Subaie S, Amiri hospital, Gulf street, Kuwait city, Kuwait, Tel +(965)99993823, Fax +(965)22464727, Email Dr_saud_@hotmail.com

Received: March II, 2017| Published: May 04, 2017
Abbreviations: LSG, laparoscopic sleeve gastrectomy; NSAID, non-steroidal anti-inflammatory drug; PPI, proton pump inhibitor; CT, computed tomography; PDS, polydioxanone suture; TPN, total parenteral nutrition

\section{Introduction}

The prevalence of obesity has been on a rapid rise in conjunction with the associated comorbidities, and the country of Kuwait is no exception. Kuwait is currently ranked the fourth 'most obese' country on the planet. ${ }^{1}$ Surgical intervention is the only effective treatment for obesity in terms of excess weight loss and subsequent improvement of comorbidities. ${ }^{2,3}$ Of the numerous bariatric procedures, the Rouxen-Y gastric bypass has proved to be effective with reproducible and statistically significant results. In parallel to the emergence of 'high-volume' bariatric surgeons, a variety of well-documented longterm complications also surfaced, such as cholelithiasis, internal hernias, dumping syndrome, short bowel syndrome, and nutritional deficiencies. In this case report, we are going to discuss a complication that we encountered in a young lady approximately lyear after Rouxen-Y gastric bypass.

\section{Case presentation}

A 25-year-old woman presented with a history of controlled hypothyroidism, Laparoscopic Sleeve Gastrostomy (LSG) in 2012, and a combined Roux-en-Y gastric bypass and cholecystectomy in 2015. She presented to one of our regional hospital surgical facilities in 2016 with a 1-day history of diffuse, non-radiating, constant, and colicky abdominal pain associated with anorexia and constipation. On examination, she was vitally stable, her abdomen was grossly distended and severely tender with evidence of guarding, and her bowel sounds were absent on auscultation. Subsequently, a Computed Tomography (CT) scan of the abdomen was done, revealing a hiatal hernia of the bypassed stomach pouch with evidence of dilated jejunal and proximal ileal loops reaching $5 \mathrm{~cm}$ in diameter (Figure 1), and free extraluminal air locules were seen in the right side of the upper abdomen and perihepatic region (Figure 2), along with mild free intra-abdominal free fluid and diffuse fat stranding (Figure 3). She was admitted as a case of acute abdomen and small bowel obstruction secondary to bowel perforation; she was immediately booked for diagnostic laparoscopy.

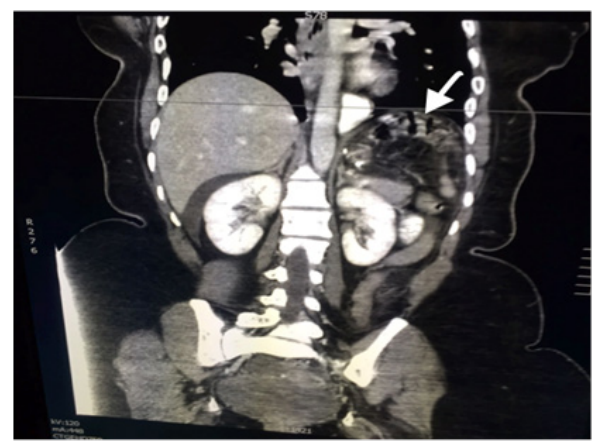

Figure I CT scan of the abdomen revealing a hiatal hernia of the bypassed stomach pouch with evidence of dilated jejunal and proximal ileal loops reaching $5 \mathrm{~cm}$ in diameter (arrows).

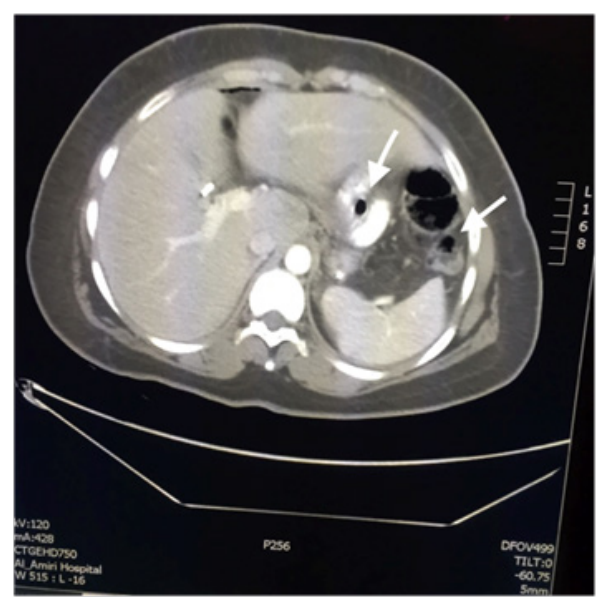

Figure $2 \mathrm{CT}$ scan showing a coronal section of the upper abdomen showing free air locules (arrows) representing pneumoperitoneum at perihepatic region. 


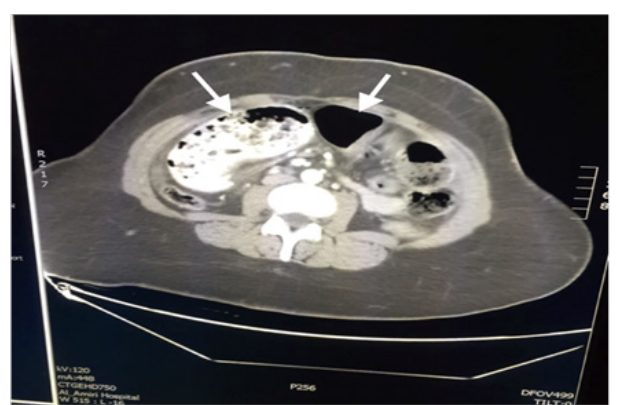

Figure 3 CT scan showing a coronal section of the mid abdomen showing dilated jejunal and upper ileal loops (arrows) with evidence of mild free intraabdominal free fluid and diffuse fat stranding.

Intraoperativley, two clear perforations were seen at the enteroenteric anastomosis with a large amount of pus in the pelvis in addition to small bowel content. Both perforations were closed under vision with Polydioxanone (PDS) sutures, and an omental patch was utilized for the defect (Figure 4). Copious irrigation was done with two drains inserted in the pelvis. The initial postoperative period was unremarkable, with the patient only complaining of mild abdominal tenderness around the port sites. Eventually, Total Parenteral Nutrition (TPN) was halted and she was subsequently started on oral feeding with a gradual escalation; she was then discharged, and she had an uneventful outpatient follow-up.

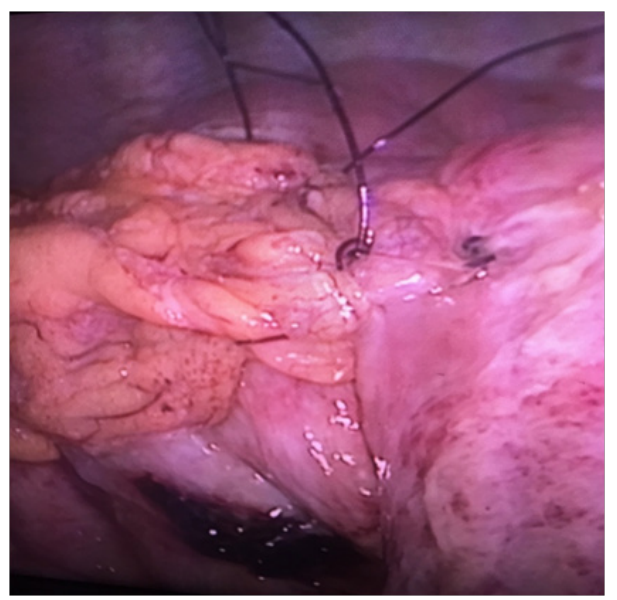

Figure 4 Laparoscopic view of perforations at the entero-enteric anastomosis closed with PDS sutures and omental patch.

\section{Discussion}

Marginal entero-enteric ulcers are a well-documented complication of Roux-en-Y gastric bypass, with an incidence between $0.6 \%$ and $16 \%{ }^{4,5}$ The majority of the published reports regarding post-gastric bypass marginal ulceration are retrospective; therefore, only symptomatic patients have been investigated, and the data we found from such studies are limited. A prospective study of 441 patients who underwent gastric bypass with endoscopy, followed up 1 month and then 1-2years after surgery, revealed that the incidence of early marginal ulceration within 1 month is $4 \%$ to $12 \%$; however, the incidence of late marginal ulceration after 1year has an incidence of $<1 \%{ }^{5}$ Predisposing factors of early and late marginal ulceration are believed to differ between patients without clear documentation in the literature. The exact mechanism of ulceration is not well established, but multiple potential etiological factors have been proposed. This patient, by consensus, is considered to have experienced a "late" anastomotic ulcer, which typically occurs 1year after surgery. Previous studies have explored increased gastric acid production, Non-steroidal Anti-Inflammatory Drug (NSAID) use, existing comorbidities, preoperative BMI, postoperative staple line bleeding, smoking, and preoperative $\mathrm{H}$. pylori infection as potential contributing factors to this phenomenon. ${ }^{6,7}$ In this case specifically, it was brought to our attention by the patient during her presentation that she was noncompliant to the recommended Proton Pump inhibitor (PPI) regimen, which in our regional hospital consists of omeprazole $40 \mathrm{mg}$ twice daily. In addition, she was 'regularly' ingesting NSAIDS for a recurrent lumbar muscular spasm. In this case study, we explore proposed etiological factors to an entero-enteric anastomotic site marginal ulcer, specifically, the synergistic effects of the former.

H. Pylori, a possible causative factor that has been extensively studied in bariatric surgery, will be mentioned for the possibility of recurrence and its documented effects on mucosal integrity. In a retrospective study of 260 patients who underwent laparoscopic gastric bypass in 2007, it was concluded that H. pylori infection, although adequately treated, was twice as common among the patients who had marginal ulceration (32\%) as among those patients who did $\operatorname{not}(12 \%)(\mathrm{p}=0.02){ }^{8}$

We presented the case of a 25-year-old woman who underwent LSG for morbid obesity, followed by a gastric bypass, because of weight gain and reflux, and developed two marginal ulcerations at the entero-enteric anastomosis because of her noncompliance with initial postoperative PPI prophylactic regiment and NSAID use for analgesia. Her ulceration was complicated with two perforations at the entero-enteric anastomosis, which was treated with revision surgery and primary repair plus omental patching of both perforations. The patient was subsequently discharged with an unremarkable outpatient follow-up. Factors related to marginal ulceration include NSAID usage, smoking, prolonged operation time, large pouch size, the use of non-absorbable sutures, and noncompliance with PPI usage. Time of presentation may vary but is usually delayed after 1year. Revision surgery is an option for patients who present with marginal ulceration associated with fistulation, chronic anemia, and perforation. However, most cases are treated medically with PPI.

Thus, because the number of bariatric surgeries, especially gastric bypass, is on the rise, the number of reported cases with such complications is also rising. However, there is no solid evidence as to what the risk factors are, the classical signs and symptoms, recommendations on prophylaxis, and management of marginal ulcerations after Roux-en-Y gastric bypass. This study serves as an invitation to focus on collecting data regarding long-term complications and subsequent management of patients undergoing laparoscopic Roux-en-Y gastric bypass.

\section{Acknowledgements}

We would like to express our sincere gratitude to Dr. Taleb Juma, chairman of department of surgery at Amiri hospital, for his guidance and valuable support throughout the course of this paper work. We acknowledge with a deep sense of gratitude, the support received from our operating theatre nurse staff to provide all the materials needed for this paper.

\section{Conflict of interest}

The author declares no conflict of interest.

\section{References}

1. Kuwait ranks No. 4 in global obesity - 'More than half of women in Kuwait overweight' 
2. Christou Nicolas, Sampalis John S, Liberman Moishe, et al. Surgery Decreases Long-term Mortality, Morbidity, and Health Care Use in Morbidly Obese Patients. Annals of surgery. 2004;240(3):416-423.

3. Sapala JA, Wood MH, Sapala MA, et al. Marginal ulcer after gastric bypass: a prospective 3-year study of 173 patients. Obes Surg. 1998;8:505-516.

4. Rasmussen JJ, Fuller W, Ali MR. Marginal ulceration after laparoscopic gastric bypass: an analysis of predisposing factors in 260 patients. Surg Endosc. 2007;21(7):1090-1094.

5. Dallal RM, Bailey LA. Ulcer disease after gastric bypass surgery. Surg Obes Relat Dis. 2006;2(4):455-459.
6. Capella JF, Capella RF. Staple disruption and marginal ulceration in gastric bypass procedures for weight reduction. Obes Surg. 1996;6(1):4449 .

7. Azagury D, Abu Dayyeh B, Greenwalt I, et al. Marginal ulceration after Roux-en-Y gastric bypass surgery: characteristics, risk factors, treatment, and outcomes. Endoscopy. 2011;43(11):950-954.

8. Rasmussen JJ, Fuller W, Ali MR. Marginal ulceration after laparoscopic gastric bypass: an analysis of predisposing factors in 260 patients. Surg Endosc. 2007;21(7):1090-1094. 